\title{
The Properties of Near-Infrared Diffuse Interstellar Bands
}

\author{
M. G. Rawlings ${ }^{1}$, A. J. Adamson ${ }^{2}$, B. J. McCall ${ }^{3}$ and T. H. Kerr ${ }^{4}$ \\ ${ }^{1}$ National Radio Astronomy Observatory, \\ 520 Edgemont Road, Charlottesville, VA 22903, U. S. A. \\ email: mrawling@nrao.edu \\ ${ }^{2}$ Gemini Observatory, 670 N. A'ohoku Place, Hilo, Hawaii, U. S. A. \\ email: aadamson@gemini.edu \\ ${ }^{3}$ Departments of Chemistry and Astronomy, University of Illinois, \\ Urbana, IL 61801, U. S. A. \\ email: bjmccall@illinois.edu \\ ${ }^{4}$ Joint Astronomy Centre, 660 N. A'ohoku Place, Hilo, Hawaii 96720, U. S. A. \\ email: t.kerr@jach.hawaii.edu
}

\begin{abstract}
In addition to the hundreds of known visual-wavelength Diffuse Interstellar Bands (DIBs), a number of DIBs in the near-infrared (NIR) are now also known to exist. We present here high-resolution UKIRT echelle spectroscopy of two of the NIR DIBs toward sightlines exhibiting a range of visual extinctions. Variations in the strengths and profile shapes of the bands are considered in the context of known properties of the narrow DIBs at visual wavelengths.
\end{abstract}

Keywords. ISM: lines and bands, ISM: molecules, dust,extinction

\section{Introduction}

Since their initial detection (Heger 1922), the identification of the carriers of the Diffuse Interstellar Bands (DIBs) has become one of the longest-standing problems in astrophysics, with several hundred visual DIBs now known to exist. Although the carriers are widely thought to be carbonaceous molecules/ions, an unambiguous identification for most of the bands remains elusive. One strong observational constraint on the nature of the carriers, however, is the detection of DIB substructure (e.g. Sarre et al. 1995).

In addition to the many visual DIBs, two DIBs were reported in the near-infrared (NIR) by Joblin et al. (1990), near 11797.5 and $13175 \AA$ A. These remained the longestwavelength DIBs known until the comparatively recent detection of DIBs in the 1.5 $1.8 \mu \mathrm{m}$ range by Geballe et al. (2011). We discuss here high-resolution spectroscopy of the two Joblin et al. (1990) NIR DIBs toward sightlines exhibiting a range of visual extinctions $\left(A_{V}\right)$, and review the band properties in the context of other known DIBs.

\section{Target Sources}

The target sources observed were chosen to reflect a range of visual extinctions:

(a) Several high-extinction diffuse medium sightlines toward both the Galactic Centre (GC) and Cygnus: The list includes the prototypical high-extinction sightline towards the hypergiant Cyg OB2 No. 12, and several of the early-type Stephenson star sightlines (Rawlings et al. 2003, Stephenson 1992). These exhibit both high diffuse ISM extinction $\left(A_{V}=6.6-15.8 \mathrm{mag}\right)$ and strong visual DIBs.

(b) $\mu$ Sgr: This bright target has strong, substructured visual DIBs (Kerr et al. 1996) and was chosen as a "single cloud" sightline in order to characterize intrinsic DIB profiles. 

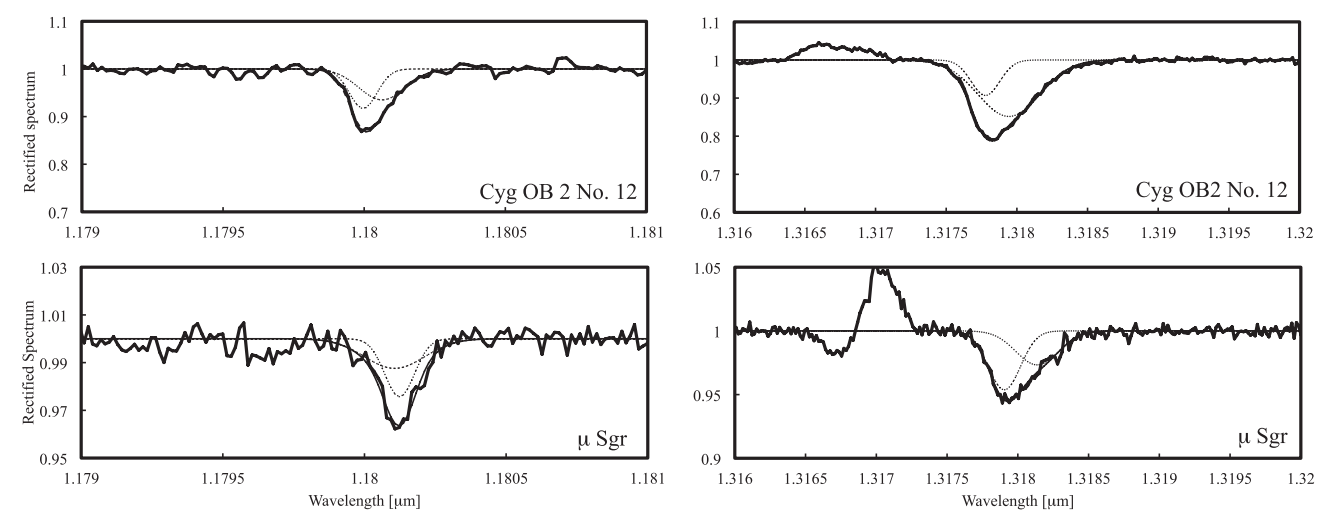

Figure 1. Examples of NIR DIBs profile fitting toward Cyg OB2 No. 12 (upper panels) and $\mu$ Sgr (lower panels). The heavy solid lines are the reduced spectra, the light dashed lines are the individual Gaussian components, and the light solid line is the overall fit.

\section{Observations and Data Reduction}

Observations took place on 2008 August 9 - 21 UT using the United Kingdom Infrared Telescope (UKIRT), Hawaii and the echelle grating on CGS4. Weather was clear for almost the entire period. A 1-pixel width slit (0.6") was used. Slit position angles for each target were chosen so as to avoid nearby field stars. $2 \times 2$ sampling was used. Observations were acquired in non-destructive readout mode using a standard 9.6" nod along the slit. Observations of the $13175 \AA$ DIB were conducted with a wavelength coverage of $1.3135-1.3215 \mu \mathrm{m}$. Observations of the $11797.5 \AA$ DIB were conducted with a wavelength coverage of $1.1762-1.1833 \mu \mathrm{m}$. The effective spectral resolution was $\sim 37000$. Integration times were adjusted in order to avoid saturation, with the aim of achieving a continuum signal-to-noise $(\mathrm{s} / \mathrm{n})$ ratio of at least 100 on all of the targets (except for $\mu \mathrm{Sgr}$ ) in order to obtain a $\sim 5 \sigma$ or better detection of the DIBs. A target $\mathrm{s} / \mathrm{n}$ ratio of $\sim 500$ was assigned to $\mu \mathrm{Sgr}$, since as the token single-cloud sightline, its DIBs were expected to be comparatively weak. Airmass-matched B-type giant stars were also observed in order to permit flux calibration. Wavelength calibration for both spectral ranges was performed using known telluric absorption features.

The data were reduced with the STARLINK software suite using standard techniques. The science target spectra were registered with suitable standard-star spectra, and the ratios of science target/standard star pairs were taken in order to remove telluric absorption effects. For cases lacking good airmass matches, other observed standard stars were tried and/or suitable logarithmic rescaling of the standard star spectrum (Rawlings et al. 2003) was performed to optimize telluric cancellation.

\section{Discussion}

\section{DIBs Profile Characteristics}

The two observed NIR DIBs can be well fitted by no more than two Gaussians in all cases. As shown in the upper panels of Fig. 1, the use of a second Gaussian is often necessary due to the presence of long-wavelength wings seen in both bands for a number of the sightlines. Similar profile shapes have previously been seen in multiple narrow visual-wavelength DIBs. Towards $\mu$ Sgr, the same behaviour is observed in the 13175 $\AA$ DIB profile, but not in the (relatively weak) $11797.5 \AA$ DIB (Fig. 1, lower panels). 

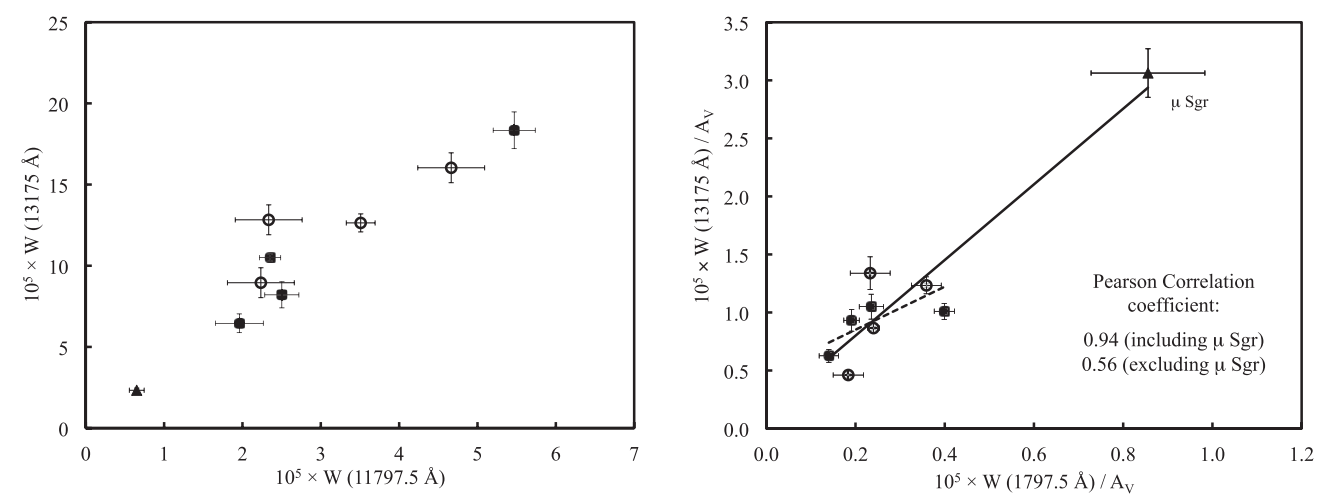

Figure 2. The strengths of the two DIBs, as measured (left) and per unit extinction (right). In both panels, filled triangles represent $\mu$ Sgr, filled circles represent sources with high Galactic longitudes (nearer Cygnus) and open circles represent sources with low Galactic longitudes (closer to the Galactic Centre). The error bars reflect the root mean square noise on the spectra.

Neither of the observed DIBs shows complex substructure at the spectral resolution used here $(\sim 37000)$.

Correlation Analysis

Figure 2 illustrates the relative strengths of the two DIBs. The left panel shows the measured DIB strengths, and although these do appear to correlate with each other, the plot is heavily influenced by the general tendency of DIB strengths to increase with diffuse medium extinction. The right panel shows the DIB strengths normalized by $A_{V}$. The Pearson correlation coefficient for the sources with measurements of both DIBs was 0.94. Previous observations at visual wavelengths, however, indicate that DIBs toward $\mu$ Sgr are unusually strong per unit $A_{V}$, and hence may not be representative of DIB strengths elsewhere. A recalculation of the correlation coefficient for the dataset with the $\mu$ Sgr data point excluded yields a value of 0.56 . As with the visual DIBs toward these stars, we detect no population distinction between the low- and high-longitude sightlines.

\section{Future Work}

Most of the high extinction sources observed here exhibit strong DIBs at other wavelengths, as well as other interstellar spectroscopic features (e.g. the $3.4 \mu \mathrm{m}$ dust absorption feature). The existence of possible (anti-)correlations between these NIR DIBs and other DIBs and organics (e.g. $3.4 \mu \mathrm{m}$ ) will therefore be investigated in the same objects.

\section{References}

Geballe, T. R., Najarro, F., Figer, D. F., Schlegelmilch, B. W., \& del a Fuente, D. 2011, Nature, 479,200

Heger, M. L. 1922, Lick Observatory Bulletin, 10, No. 337, 146

Hobbs, L. M., York, D. G., Snow, T. P., Oka, T., Thorburn, J. A., Bishof, M., Friedman, S. D., McCall, B. J., Rachford, B., Sonnentrucker, P., \& Welty, D. E. 2008, ApJ, 680, 1256

Joblin, C., D'Hendecourt, L., Leger, A., \& Maillard, J. P. 1990, Nature, 346, 729

Kerr, T. H., Hibbins, R. E., Miles, J. R., Fossey, S. J., Somerville, W. B., \& Sarre, P. J. 1996, MNRAS, 283L, 105

Rawlings, M. G., Adamson, A. J., \& Whittet, D. C. B. 2003, MNRAS, 341, 1121

Sarre, P. J., Miles, J. R., Kerr, T. H., Hibbins, R. E., \& Somerville, W. B. 1995, MNRAS, 277L, $41 \mathrm{~S}$

Stephenson, C. B. 1992, AJ, 103, 263 